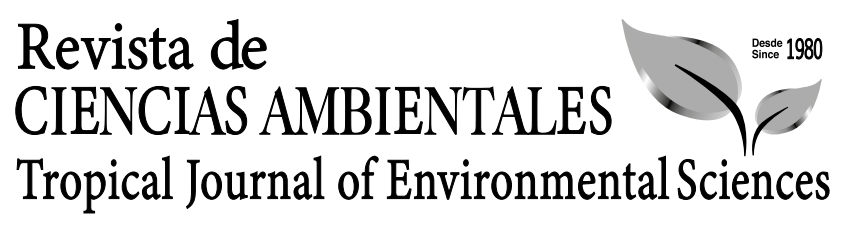

Revista de Ciencias Ambientales (Trop J Environ Sci)

e-ISSN: 2215-3896

(Enero-Junio, 2021) . Vol 55(1): 32-51

DOI: https://doi.org/10.15359/rca.55-1.2

Open Access: www.revistas.una.ac.cr/ambientales

e-mail: revista.ambientales@una.ac.cr

Solano-Salmerón J., Fonseca-González W., Ugalde-Alfaro S. y Alice-Guier F.

\title{
Impacto sobre el cambio climático del ciclo de vida de las tarimas de madera elaboradas en la región Huetar Norte de Costa Rica
}

\author{
Climate change impact of the life cycle of wooden pallets made in the Northern \\ Huetar region in Costa Rica
}

\author{
John Solano Salmerón ${ }^{1}$, William Fonseca González², Sebastián Ugalde Alfaro \\ Federico Alice Guier ${ }^{4}$
}

[Recibido: 25 de abril 2020, Aceptado: 28 de agosto 2020, Corregido: 8 de octubre 2020, Publicado: 1 de enero 2021]

\section{Resumen}

[Introducción]: El Análisis de Ciclo de Vida (ACV) es una metodología robusta y reconocida para determinar la magnitud de los impactos ambientales de un producto. [Objetivo]: El objetivo de la investigación fue evaluar el impacto en la categoría de cambio climático de las tarimas de madera. [Metodología]: Los límites del sistema van desde la preparación del terreno para establecer las plantaciones, hasta la puerta de llegada a la empacadora de fruta. Se evaluaron dos unidades funcionales: a) una tarima con dimensiones comerciales para la manipulación y trasiego de productos agrícolas y b) un metro cúbico de madera para producción de tarimas. La temporalidad de los datos comprendió el periodo 2014-2017 y la de los resultados del Análisis de Ciclo de Vida un ciclo de rotación de 12 años para plantaciones de melina (Gmelina arborea Roxb). Se evaluaron dos sistemas de producto: con biomasa en el tratamiento térmico y con gas LP. [Resultados]: El primero presentó un impacto potencial levemente menor. En ambos casos, la combustión de diésel asociada al transporte de madera rolliza fue la etapa más intensa en emisiones, libera cerca de $1.61 \mathrm{~kg} \mathrm{CO}_{2}$-eq tarima ${ }^{-1}$ y $43.22 \mathrm{~kg} \mathrm{CO}_{2}$-eq m${ }^{-3}$. El diésel, en todas las etapas del ciclo de vida, es la principal fuente de emisión de gases de efecto invernadero, ya que libera $2.31 \mathrm{~kg} \mathrm{CO}_{2}$-eq tarima ${ }^{-1} \mathrm{y}$ $86.57 \mathrm{~kg} \mathrm{CO}_{2}$-eq m${ }^{-3}$. [Conclusiones]: Las oportunidades de mejora en el desempeño ambiental del producto se encuentran asociadas al uso de vehículos de carga más eficientes y la reducción de la distancia de acarreo entre las plantaciones forestales y la planta de aserrío.

Palabras clave: Cambio climático; Evaluación de Impacto de Ciclo de Vida (EICV); huella de carbono de producto (HCP); Inventario de Ciclo de Vida (ICV); productos biogénicos; productos de madera recolectada (PMR); productos forestales.

1 Ingeniero en ciencias forestales, Ente Costarricense de Acreditación (ECA), j.solano@eca.or.cr; https://orcid.org/0000-0001-9487-112X

2 Académico, Escuela de Ciencias Ambientales e Instituto de Investigación y Servicios Ambientales, Universidad Nacional, Costa Rica; wfonseca@una.ac.cr; https://orcid.org/0000-0002-4546-9035

3 Consultor, Gestor de Silvicultura, Industria y Comercio, Oficina Nacional Forestal, Costa Rica; sugalde@oficinaforestalcr.org; https://orcid.org/0000-0002-8620-1107

4 Académico, Escuela de Ciencias Ambientales, Universidad Nacional, Costa Rica; federico.alice.guier@una.ac.cr; https://orcid.org/0000-0002-6155-0562

繁

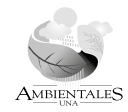




\section{Revista de CIENCIAS AMBIENTALES Tropical Journal of Environmental Sciences}

Revista de Ciencias Ambientales (Trop J Environ Sci) e-ISSN: 2215-3896 (Enero-Junio, 2021) . Vol 55(1): 32-51 DOI: https://doi.org/10.15359/rca.55-1.2 Open Access: www.revistas.una.ac.cr/ambientales e-mail: revista.ambientales@una.ac.cr Solano-Salmerón J., Fonseca-González W., Ugalde-Alfaro S. y Alice-Guier F.

\begin{abstract}
[Introduction]: Life Cycle Analysis is a powerful and well-recognized methodology to determine a product's environmental impact [Objective]: The aim of this research was to evaluate the impact of wooden pallets on the climate change category. [Methodology]: The scope of the system ranges from land preparation to establish the plantations to the arrival gate at the fruit packing house. Two functional units were assessed: a) a pallet with commercial dimensions for handling of agricultural products and b) a cubic meter of wood to produce pallets. The study included data from the 2014-2017 period, and life cycle results included a whole rotation equivalent to 12 years for the gmelina (Gmelina arborea Roxb) plantations. Two product systems were evaluated: using biomass in the heat treatment and using LP gas. [Results]: The former presented a slightly smaller potential impact. In both cases, the diesel combustion associated with roundwood transport was the most emissions intense stage, releasing approximately $1.61 \mathrm{~kg} \mathrm{CO}_{2}$-eq pallet ${ }^{-1}$ and $43.22 \mathrm{~kg} \mathrm{CO}_{2}$-eq m$~^{-3}$. Diesel is the primary source of greenhouse gas emissions in all life cycle stages, releasing $2.31 \mathrm{~kg} \mathrm{CO}_{2}$-eq pallet ${ }^{-1}$ and $86.57 \mathrm{~kg} \mathrm{CO}_{2}$-eq m${ }^{-3}$. [Conclusions]: Opportunities to improve the product's environmental performance include using more efficient loading vehicles and reducing the distance between forest plantations and the sawmill.
\end{abstract}

Keywords: Biogenic products; climate change; forest products; Harvested Wood Products (HWP); Life Cycle Impact Assessment (LCIA); Life Cycle Inventory (LCI); Product Carbon Footprint (PCF).

\title{
1. Introducción
}

El trasiego de mercancías a nivel mundial se realiza con tarimas; cada día, este producto cobra mayor importancia porque agiliza las labores de manipulación, reduce costos y mejora la eficiencia en las labores de logística, dado que permite mover mayor cantidad de producto en una sola operación (Elia y Grazia, 2015). Las tarimas o pallets son plataformas rígidas fácilmente manejables y movilizables por medios mecánicos que se utilizan para colocar, almacenar, apilar, maniobrar y transportar mercancías embaladas o sueltas. Para su elaboración se utilizan materiales como madera, metal, plástico y otros; las de madera son las más comunes (Elia y Grazia, 2015). Se elaboran en diferentes diseños, de acuerdo con los requerimientos de los productos a colocar. Se estima que en el pasado han existido hasta 2000 diseños (Platt y Hyde, 1997).

En Estados Unidos se estima que se producen 700 millones de tarimas por año, que llegan a formar parte de los aproximadamente 2 billones de unidades que se mantienen en circulación (Mazeika, 2011). En la Unión Europea se producen entre 280 a 400 millones de unidades por año (García, Farreny, Navarro y Boschmonart, 2016; Mazeika, 2011; Niero, Di Felice y Ren, 2014).

En Costa Rica, la producción de tarimas supera 6 millones de unidades por año, con una tasa anual de crecimiento para el 2018 de $7.22 \%$. Su principal uso es la manipulación y el trasiego de productos agrícolas de exportación; en especial piña y banano (Oficina Nacional Forestal [ONF], 2018). La industria de tarimas en el país se abastece en su mayoría de especies de madera nacional y minoritariamente de madera de pino chileno. Se estima que esta industria consume más del $42 \%$ de la madera procesada en el país, un volumen superior a 433 mil metros cúbicos de madera rolliza por año (ONF, 2018).

\begin{tabular}{|c|c|c|c|c|}
\hline 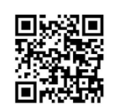 & (c) (i) () () & $\underset{\text { AMBENENALIES }}{\infty}$ & $\frac{1 \%}{2 \%}$ & 33 \\
\hline
\end{tabular}




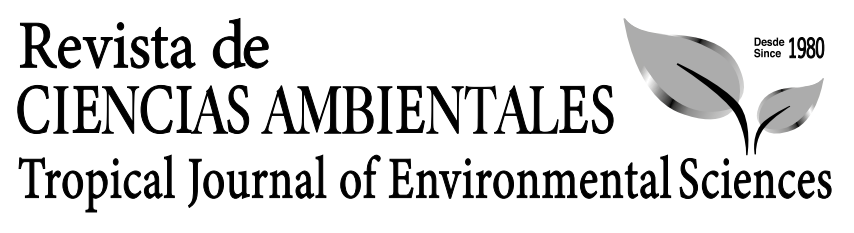

Revista de Ciencias Ambientales (Trop J Environ Sci) e-ISSN: $2215-3896$ (Enero-Junio, 2021) . Vol 55(1): 32-51 DOI: https://doi.org/10.15359/rca.55-1.2 Open Access: www.revistas.una.ac.cr/ambientales e-mail: revista.ambientales@una.ac.cr Solano-Salmerón J., Fonseca-González W., Ugalde-Alfaro S. y Alice-Guier F.

Las tarimas, como producto de madera, presentan un elevado potencial de mitigación del cambio climático, tanto en la oferta como en la demanda. En la oferta, a través de la remoción y almacenamiento de carbono en la biomasa de los ecosistemas forestales y en productos. Por ejemplo, un metro cúbico de madera puede almacenar entre 678.32 y $734 \mathrm{~kg} \mathrm{CO}_{2}$-eq m${ }^{-3}$ y una tarima alrededor de $8.2 \mathrm{~kg}$ de $\mathrm{CO}_{2}$-eq (García et al., 2016; Klein, Wolf, Schulz y Weber, 2015; Puettmann, Oneil y Bergman, 2013). Por otro lado, un aumento en la producción forestal no necesariamente significa un incremento en las emisiones de gases de efecto invernadero (GEI) ya que se pueden tomar medidas que reduzcan la cantidad de GEI por unidad de producto fabricado a partir de los resultados del ACV. (IPCC, 2014). Para tarimas, la información publicada sobre la cantidad de emisiones por unidad de producto es escasa. Sin embargo, se han encontrado valores de 1.57 hasta $20.05 \mathrm{~kg} \mathrm{CO}_{2}$-eq tarima ${ }^{-1}$ según la región, el sistema de producción y el abordaje metodológico (Anil, 2010; Niero et al., 2014; García et al., 2016, Solano, 2018). En la demanda, principalmente por la capacidad de sustituir o reemplazar bienes más intensos en emisiones de gases de efecto invernadero (FAO, 2010; IPCC, 2014).

A nivel mundial, la industria de tarimas demanda una considerable cantidad de madera como principal materia prima; así como otros recursos naturales. Una forma robusta y de gran reconocimiento científico para determinar la magnitud en la cual este producto contribuye a mitigar el cambio climático es a través de los estudios de análisis de ciclo de vida (ACV). El ACV es un inventario de entradas y salidas del proceso productivo, por medio del cual es posible evaluar los impactos ambientales potenciales de un sistema del producto a lo largo de su ciclo de vida. Es decir, a lo largo de su cadena de producción, desde la extracción de las materias primas, hasta la disposición final del producto, pasando por las etapas de transporte, manufactura y distribución (Heinimann, 2012; INTE/ISO, 2007ab; Life Cycle Initiative, 2017).

El ACV tiene como finalidad evaluar la magnitud de los impactos ambientales potenciales de un sistema del producto para diferentes propósitos; entre ellos: i- identificar oportunidades de mejora en su desempeño ambiental, ii- aportar información para la planificación estratégica, iii- establecer aseveraciones comparativas entre productos que desempeñan una misma función, entre otras (INTE/ ISO 2007ab). Debido a los compromisos internacionales adquiridos por el país en materia de cambio climático (Costa Rica/MINAET, 2009), al alto porcentaje de madera que consume la industria de tarimas a nivel nacional y al potencial de mitigación del cambio climático, se desarrolló esta investigación cuyo objetivo fue determinar el impacto ambiental potencial de la producción de tarimas de madera elaboradas en la región Huetar Norte, evaluando la categoría de impacto cambio climático y en busca de identificar oportunidades de mejora en su desempeño ambiental.

\section{Metodología}

\section{1 Área de estudio, límites del sistema del producto y unidad funcional}

El área de estudio fue la región Huetar Norte de Costa Rica, específicamente los cantones Guatuso, Los Chiles, San Carlos y Upala (Figura 1). Esta región se caracteriza por su gran

\begin{tabular}{|c|c|c|c|c|c|}
\hline 啝知 & (c) (i) (2) (2) & 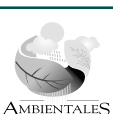 & 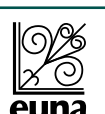 & $\frac{\text { UNA }}{\frac{\text { UNNESIDAD }}{\text { INACINONAL }}}$ & 34 \\
\hline
\end{tabular}




\section{Revista de \\ CIENCIAS AMBIENTALES \\ Tropical Journal of Environmental Sciences}

Revista de Ciencias Ambientales (Trop J Environ Sci) e-ISSN: 2215-3896 (Enero-Junio, 2021) . Vol 55(1): 32-51 DOI: https://doi.org/10.15359/rca.55-1.2 Open Access: www.revistas.una.ac.cr/ambientales e-mail: revista.ambientales@una.ac.cr Solano-Salmerón J., Fonseca-González W., Ugalde-Alfaro S. y Alice-Guier F.

desarrollo forestal, atribuido a diferentes factores, entre ellos: contiene una considerable extensión de terreno con plantación forestal de carácter comercial, alberga la mayor cantidad de aserraderos del país y es la zona que genera el mayor aporte de madera nacional (Sistema Nacional de Áreas de Conservación [SINAC] 2011; SINAC, SIREFOR y MINAE 2013).
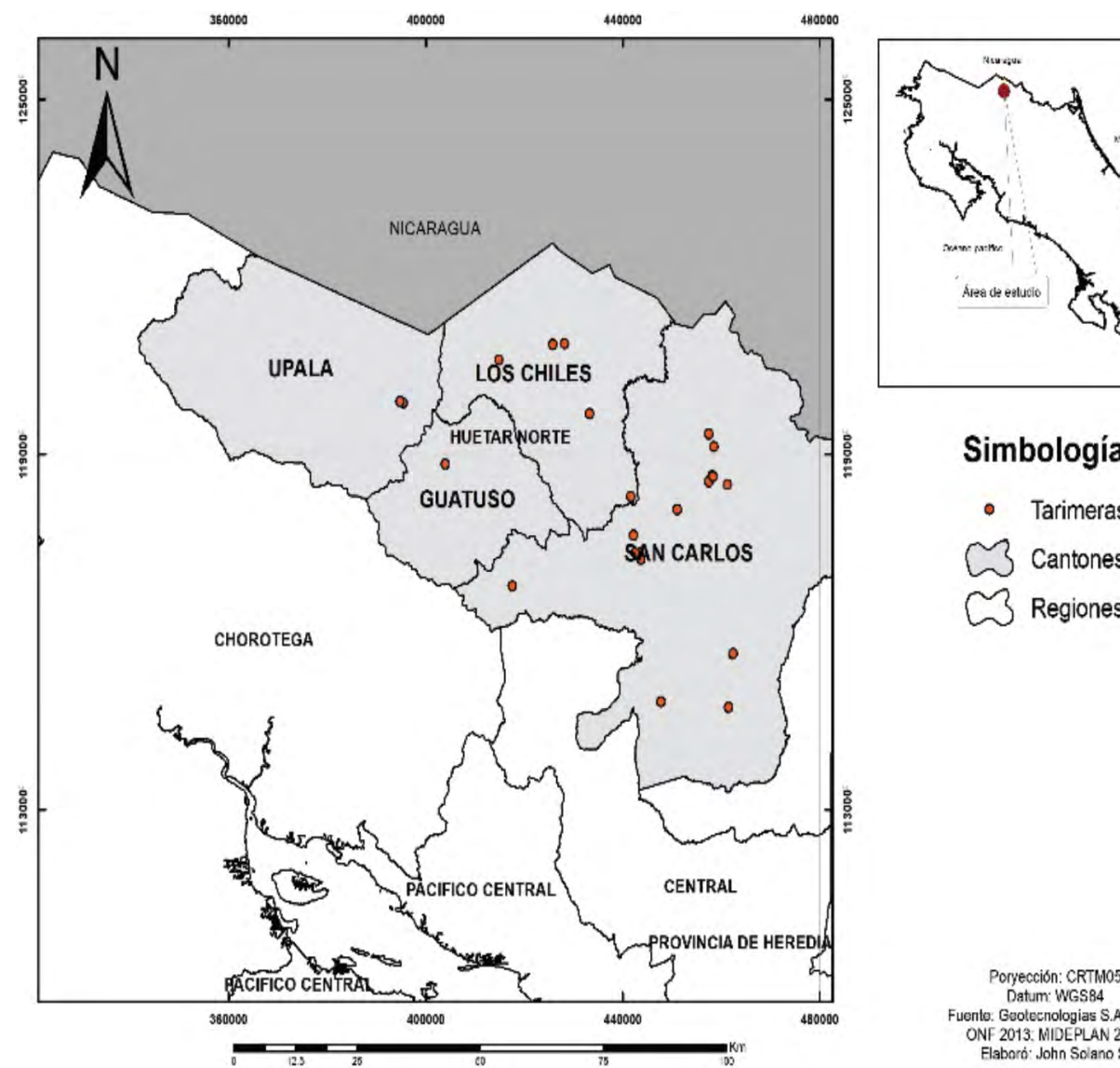

Figura 1. Ubicación geográfica del área de estudio: Región Huetar Norte, Costa Rica.

Figure 1. Geographic location of the study area: Huetar Norte Region, Costa Rica.

La región limita al norte con Nicaragua y el río San Juan, al sur con la Cordillera Volcánica Central, al oeste con la Cordillera de Guanacaste y la Cordillera de Tilarán; y al este con el río Chirripó. Tiene una extensión de 7419 km². Según el sistema de clasificación de zonas de vida propuesto por Holdridge se localiza en el bosque húmedo tropical, bosque muy húmedo tropical y bosque pluvial (Centro Científico Tropical [CCT] y Ministerio de Agricultura y Ganadería [MAG] 2004). El clima es lluvioso todo el año, con una leve disminución en febrero, marzo y abril, la precipitación media ronda los 1 700-4 $400 \mathrm{~mm}$ anuales. La temperatura media anual oscila entre $18-32^{\circ} \mathrm{C}$ (Instituto Meteorológico Nacional [IMN] 2014).

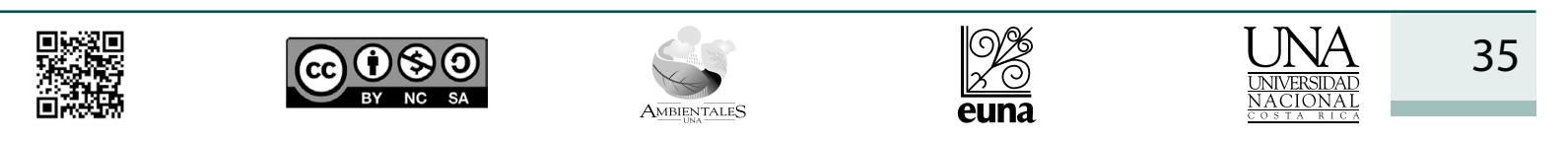




\section{Revista de \\ CIENCIAS AMBIENTALES \\ Tropical Journal of Environmental Sciences}

Revista de Ciencias Ambientales (Trop J Environ Sci)

e-ISSN: 2215-3896

(Enero-Junio, 2021) . Vol 55(1): 32-51

DOI: https://doi.org/10.15359/rca.55-1.2

Open Access: www.revistas.una.ac.cr/ambientales

e-mail: revista.ambientales@una.ac.cr

Solano-Salmerón J., Fonseca-González W., Ugalde-Alfaro S. y Alice-Guier F.

Los límites del sistema del producto en estudio comprenden las etapas de: i- preparación del terreno, ii- establecimiento de las plantaciones, iii) manejo silvicultural, iv) transporte, v) aserrío, vi) ensamblaje de las tarimas, vii) acabado y vii) distribución (Figura 2). Las etapas de uso y disposición final del producto no fueron tomadas en cuenta en este análisis, tampoco las emisiones asociadas a infraestructura, maquinaria y equipo, dado que su aporte se supone insignificante como resultado de su relativamente largo periodo de vida útil (García et al., 2016).

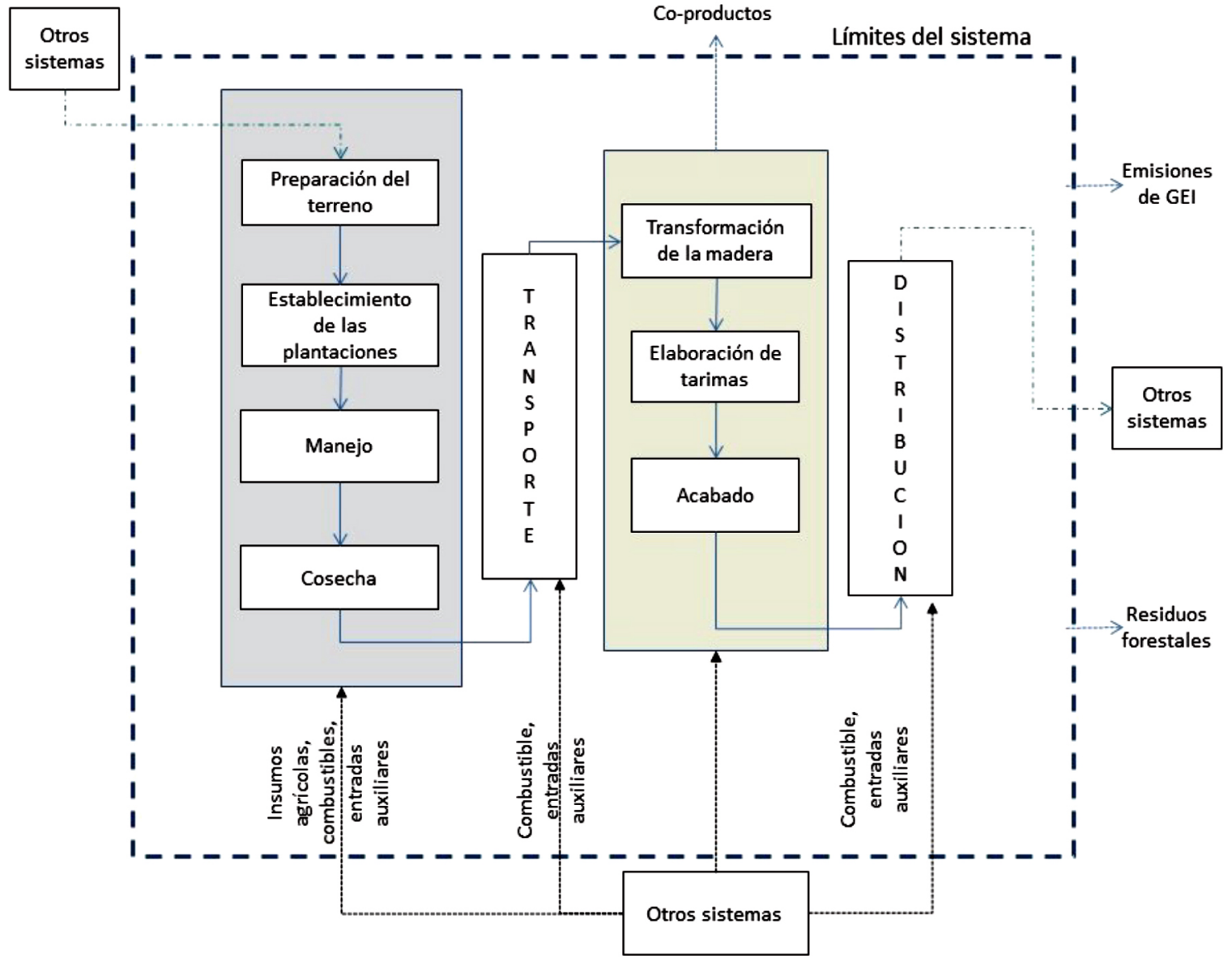

Figura 2. Límites del sistema del producto bajo estudio.

Figure 2. System limits of the product under study.

En el estudio se consideraron dos unidades funcionales. La primera, una tarima de madera con dimensiones comerciales para la manipulación y trasiego de productos agrícolas de exportación; y la segunda, un metro cúbico de madera aserrada $\left(\mathrm{m}^{3}\right)$ destinado al mismo uso.

\begin{tabular}{|c|c|c|c|c|c|}
\hline 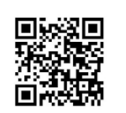 & (c) () () () & $\bigotimes_{\text {AMBEENTALS }}$ & $\begin{array}{l}\frac{9 \%}{2} \\
\frac{2}{2} \\
\text { euna }\end{array}$ & $\frac{\text { UNA }}{\frac{\text { UNIIERISAD }}{\text { UASEIONAL }}}$ & 36 \\
\hline
\end{tabular}




\section{Revista de CIENCIAS AMBIENTALES Tropical Journal of Environmental Sciences}

Revista de Ciencias Ambientales (Trop J Environ Sci) e-ISSN: 2215-3896

(Enero-Junio, 2021) . Vol 55(1): 32-51

DOI: https://doi.org/10.15359/rca.55-1.2 Open Access: www.revistas.una.ac.cr/ambientales e-mail: revista.ambientales@una.ac.cr Solano-Salmerón J., Fonseca-González W., Ugalde-Alfaro S. y Alice-Guier F.

\subsection{Inventario de ciclo de vida, temporalidad y carbono en producto}

El inventario de ciclo de vida (ICV) registra las entradas y salidas que representa al sistema del producto (Apéndice 1). En la recopilación de información se utilizaron datos primarios y secundarios según la disponibilidad de tiempo y recursos. Los datos primarios comprenden las entradas del inventario o datos de actividad; y los datos secundarios son los factores de cálculo de emisiones. En específico, los factores de emisión de gases de efecto invernadero y los potenciales de calentamiento global.

Los datos del ICV (Cuadro 1) se estimaron a través de una serie de entrevistas y aplicación de cuestionarios, se utilizó el método conocido como recuperación de rendimientos (Guevara y Murillo, 2009; Louman, Gómez y Navarro, 2006; Reiche, 1989). De esta manera, se registraron los datos de entrada a nivel de proceso unitario (Apéndice 2), grupo de procesos, a nivel de operación, grupos de operaciones, etapa del ciclo de vida o grupos de etapas; según la disponibilidad de información del entrevistado. Se realizaron 93 entrevistas distribuidas de la forma que muestra el Cuadro 2.

La temporalidad de los datos recopilados comprendió al periodo 2014-2017 y la temporalidad de los resultados del ACV representó un ciclo de rotación completo para plantaciones forestales de melina (G. arborea) equivalente a 12 años (Sage y Quirós, 2001). Con el fin de reflejar la variabilidad encontrada en los sistemas del producto, las entradas del inventario corresponden a valores promedios, ponderados según el área de plantación forestal o volumen de producción de tarimas de las empresas.

El carbono almacenado en el producto se estimó a partir del promedio ponderado considerando el porcentaje de usos de las diferentes especies forestales empleadas en la fabricación de tarimas de madera en la región (Figura 3). El cálculo se realizó a través del método indirecto, a partir del volumen, la densidad específica y la fracción de carbono de la madera (Fonseca, 2009; IPCC, 2006; Martínez y Berdasco, 2015).

Cuadro 1. Entradas del inventario de ciclo de vida (ICV) y factores de emisión utilizados para la evaluación del impacto de ciclo de vida (EICV).

Table 1. Life cycle inventory (LCI) entries and emission factors used for the Life Cycle Impact Assessment (LCIA).

\begin{tabular}{|c|c|c|c|c|c|c|c|}
\hline \multirow{2}{*}{ Entradas del ICV } & \multicolumn{2}{|c|}{$\mathrm{CO}_{2}$} & \multicolumn{2}{|c|}{$\mathrm{CH}_{4}$} & \multicolumn{2}{|c|}{$\mathrm{N}_{2} \mathrm{O}$} & \multirow{2}{*}{ Referencia } \\
\hline & Valor & Unidades & Valor & Unidades & Valor & Unidades & \\
\hline $\begin{array}{l}\text { Gasolina }{ }^{a} / \\
\text { maquinaria agrícola }\end{array}$ & 2.26 & $\mathrm{~kg} / \mathrm{L}$ & 3.27E-04 & $\mathrm{kg} / \mathrm{L}$ & $1.96 \mathrm{E}-05$ & $\mathrm{~kg} / \mathrm{L}$ & IMN, 2015 \\
\hline $\begin{array}{l}\text { Diésela / maquinaria } \\
\text { agrícola }\end{array}$ & 2.69 & $\mathrm{~kg} / \mathrm{L}$ & $3.63 \mathrm{E}-04$ & $\mathrm{~kg} / \mathrm{L}$ & $2.18 \mathrm{E}-05$ & $\mathrm{~kg} / \mathrm{L}$ & IMN, 2015 \\
\hline $\begin{array}{l}\text { Diésel/ } \\
\text { transporte de madera }\end{array}$ & 2.69 & $\mathrm{~kg} / \mathrm{L}$ & $3.63 \mathrm{E}-04$ & $\mathrm{~kg} / \mathrm{L}$ & $2.18 \mathrm{E}-05$ & $\mathrm{~kg} / \mathrm{L}$ & IMN, 2015 \\
\hline $\begin{array}{l}\text { Fertilizantes }{ }^{\mathrm{b}} / \\
\text { ha de plantación }\end{array}$ & 2.93 & $\mathrm{~kg} / \mathrm{kg}$ & $\mathrm{ND}^{\mathrm{c}}$ & $\mathrm{kg} / \mathrm{kg}$ & ND & $\mathrm{kg} / \mathrm{kg}$ & FAO, $2006^{a}$ \\
\hline $\begin{array}{l}\text { Ureab/ ha de } \\
\text { plantación }\end{array}$ & 0.73 & $\mathrm{~kg} / \mathrm{kg}$ & $\mathrm{NA}^{\mathrm{d}}$ & NA & NA & NA & $\begin{array}{l}\text { IMN, 2015; } \\
\text { IPCC, } 2006\end{array}$ \\
\hline
\end{tabular}

(cc)(3)




\section{Revista de CIENCIAS AMBIENTALES

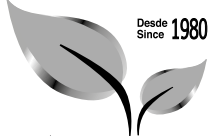 Tropical Journal of Environmental Sciences}

Revista de Ciencias Ambientales (Trop J Environ Sci) e-ISSN: 2215-3896 (Enero-Junio, 2021). Vol 55(1): 32-51 DOI: https://doi.org/10.15359/rca.55-1.2 Open Access: www.revistas.una.ac.cr/ambientales e-mail: revista.ambientales@una.ac.cr Solano-Salmerón J., Fonseca-González W. Ugalde-Alfaro S. y Alice-Guier F.

\begin{tabular}{|c|c|c|c|c|c|c|c|}
\hline \multirow{2}{*}{ Entradas del ICV } & \multicolumn{2}{|c|}{$\mathrm{CO}_{2}$} & \multicolumn{2}{|c|}{$\mathrm{CH}_{4}$} & \multicolumn{2}{|c|}{$\mathrm{N}_{2} \mathrm{O}$} & \multirow{2}{*}{ Referencia } \\
\hline & Valor & Unidades & Valor & Unidades & Valor & Unidades & \\
\hline $\begin{array}{l}\text { Nitrógeno sintético } / \\
\text { ha de plantación }\end{array}$ & NA & NA & NA & NA & 0,016 & $\mathrm{~kg} / \mathrm{kg} \mathrm{N}$ & $\begin{array}{l}\text { IMN, 2015; } \\
\text { IPCC, } 2006\end{array}$ \\
\hline $\begin{array}{l}\mathrm{Cal} \text { / } \\
\text { ha de plantación }\end{array}$ & 0.75 & $\mathrm{~kg} / \mathrm{kg}$ & NA & NA & NA & NA & IMN, 2015 \\
\hline $\begin{array}{l}\mathrm{CaCO}_{3}^{\mathrm{b}} / \\
\text { ha de plantación }\end{array}$ & 0.44 & $\mathrm{~kg} / \mathrm{kg}$ & NA & NA & NA & NA & IPCC, 2006 \\
\hline $\begin{array}{l}\text { Diésel }^{\text {a }} \text { transporte de } \\
\text { tarimas }\end{array}$ & 2.69 & $\mathrm{~kg} / \mathrm{L}$ & $1.42 \mathrm{E}-04$ & $\mathrm{~kg} / \mathrm{L}$ & $1.42 \mathrm{E}-04$ & $\mathrm{~kg} / \mathrm{L}$ & IMN, 2015 \\
\hline Lubricantes $^{\mathrm{b}}$ & 0.5101 & $\mathrm{~kg} / \mathrm{L}$ & ND & $\mathrm{kg} / \mathrm{kg}$ & ND & $\mathrm{kg} / \mathrm{kg}$ & IMN, 2015 \\
\hline Electricidad $^{\mathrm{b}}$ & $0.117^{\mathrm{e}}$ & $\mathrm{kg} / \mathrm{kWh}$ & NA & NA & NA & NA & IMN, 2015 \\
\hline $\begin{array}{l}\text { Acero }^{\mathrm{b}} \\
\text { (clavos y grapas) }\end{array}$ & 1.06 & $\mathrm{~kg} / \mathrm{kg}$ & $0.00 \mathrm{E}+00$ & $\mathrm{~kg} / \mathrm{kg}$ & $0.00 \mathrm{E}+00$ & $\mathrm{~kg} / \mathrm{kg}$ & IPCC, 2006 \\
\hline $\mathrm{GLP}^{\mathrm{b}} /$ manufactura & 1.61 & $\mathrm{~kg} / \mathrm{L}$ & $2.55 \mathrm{E}-05$ & $\mathrm{~kg} / \mathrm{L}$ & $2.55 \mathrm{E}-06$ & $\mathrm{~kg} / \mathrm{L}$ & IMN, 2015 \\
\hline $\begin{array}{l}\text { Biomasa }^{a} / \\
\text { manufactura }\end{array}$ & $0,00 \mathrm{E}+00$ & $\mathrm{~kg} / \mathrm{MJ}$ & 0.0003 & $\mathrm{~kg} / \mathrm{MJ}$ & $4.00 \mathrm{E}-06$ & $\mathrm{~kg} / \mathrm{MJ}$ & IPCC, 2006 \\
\hline
\end{tabular}

${ }^{\mathrm{a}}$ Combustión, ${ }^{\mathrm{b}}$ uso, ${ }^{\mathrm{c}} \mathrm{ND}$ : sin información, ${ }^{\mathrm{d}} \mathrm{NA}$ : no aplica, ${ }^{\mathrm{e}}$ valor en $\mathrm{CO}_{2}$-eq.

Cuadro 2. Número de personas entrevistadas por etapa del ciclo de vida.

Table 2. Number of interviewees by stage of the life cycle.

\begin{tabular}{|c|c|c|}
\hline ID & Etapa del CV & Muestra (n) \\
\hline 1 & Preparación del terreno & 19 \\
\hline 2 & Establecimiento de la plantación & 9 \\
\hline 3 & Manejo silvicultural & 12 \\
\hline 4 & Transporte & 11 \\
\hline 5 & Transformación & 10 \\
\hline 6 & Elaboración & 10 \\
\hline 7 & Acabado & 10 \\
\hline 8 & Distribución & 12 \\
\hline Total & & 93 \\
\hline
\end{tabular}

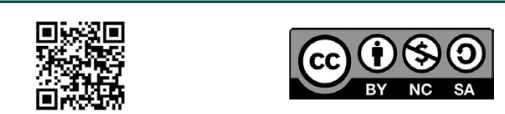




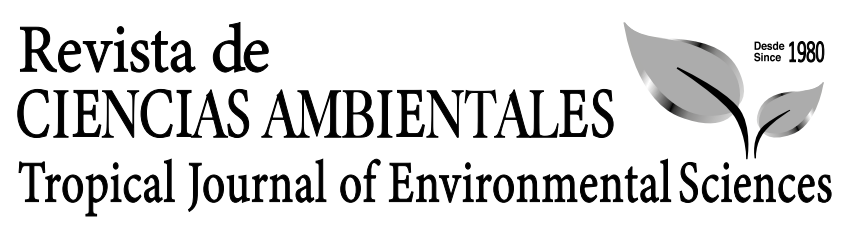

Revista de Ciencias Ambientales (Trop J Environ Sci) e-ISSN: 2215-3896 (Enero-Junio, 2021) . Vol 55(1): 32-51 DOI: https://doi.org/10.15359/rca.55-1.2 Open Access: www.revistas.una.ac.cr/ambientales e-mail: revista.ambientales@una.ac.cr Solano-Salmerón J., Fonseca-González W., Ugalde-Alfaro S. y Alice-Guier F.

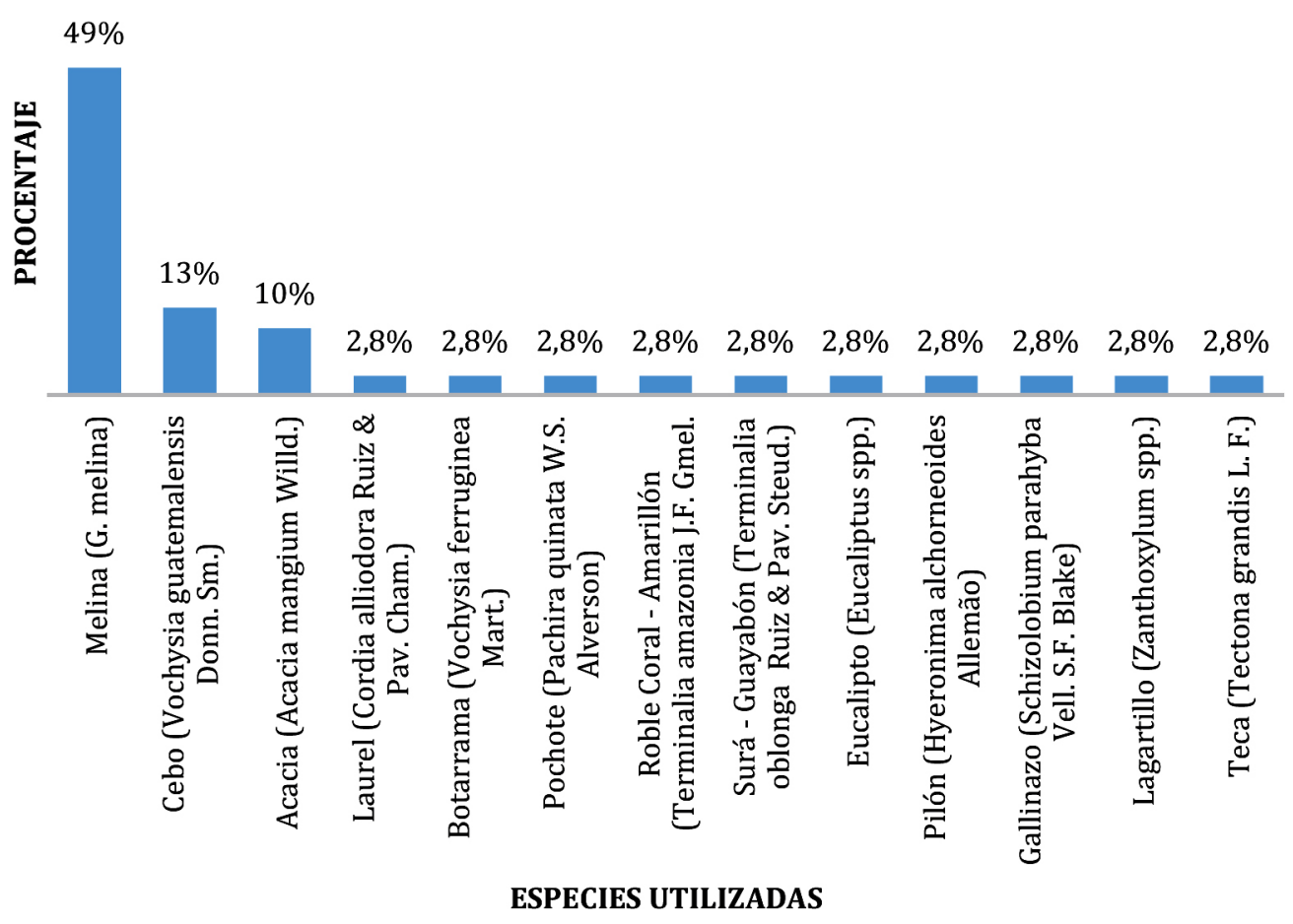

Figura 3. Porcentaje de uso de especies forestales empleadas en la fabricación de tarimas de madera. Región Huetar Norte, Costa Rica. 2014-2017.

Figure 3. Percentage of use of forest species used in the manufacture of wooden pallets. Huetar Norte Region, Costa Rica. 2014-2017.

El volumen de madera por tarima se obtuvo a partir del cubicaje de 23 tarimas, la densidad empleada fue de $456 \mathrm{~kg} \mathrm{~m}^{-3}$ (Blanco, Carpio y Muñoz, 2005; Moya et al., 2010) y la fracción de carbono de 0.42 (Cubero y Rojas, 1999; Fonseca, 2009; IPCC, 2006). Para la densidad y la fracción de carbono, las cifras indicadas corresponden al promedio ponderado según el porcentaje de uso de las especies utilizadas en la región para la producción de tarimas. El cálculo del carbono en producto se realizó a través de la Ecuación 1 (IPCC, 2006):

$$
C_{\mathrm{p}}=\left(V^{*} \beta^{*} F C\right) * 3.67(\text { E. } 1)
$$

Donde:

$C_{\text {: }}$ : reservorio de carbono en producto, $\mathrm{kg}$ de $\mathrm{CO}_{2}$ por unidad funcional.

$V:$ volumen de madera $\left(\mathrm{m}^{3}\right)$.

$\beta$ : densidad de la madera $\left(\mathrm{kg} / \mathrm{m}^{3}\right)$.

FC: fracción de carbono y la constante 3.67 (relación molecular C- $\mathrm{CO}_{2}$ ).

\begin{tabular}{|c|c|c|c|c|c|}
\hline 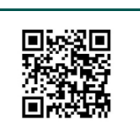 & (c) (i) () (2) & 8 & $\frac{2 \%}{20}$ & 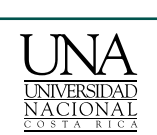 & 39 \\
\hline
\end{tabular}




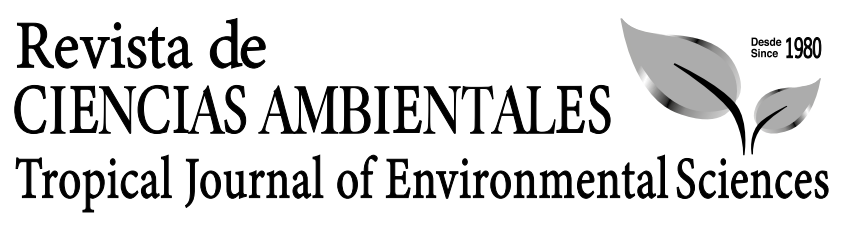

Revista de Ciencias Ambientales (Trop J Environ Sci) e-ISSN: $2215-3896$ (Enero-Junio, 2021) . Vol 55(1): 32-51 DOI: https://doi.org/10.15359/rca.55-1.2 Open Access: www.revistas.una.ac.cr/ambientales e-mail: revista.ambientales@una.ac.cr Solano-Salmerón J., Fonseca-González W., Ugalde-Alfaro S. y Alice-Guier F.

\subsection{Evaluación del impacto de ciclo de vida (EICV) de las tarimas de madera}

Este estudio se concentró en evaluar únicamente el impacto de ciclo de vida (EICV) en la categoría de cambio climático. Para ello, se siguieron las directrices del Grupo Intergubernamental de Expertos en Cambio Climático [IPCC] (2006). En forma general, el cálculo se realizó aplicando la ecuación 2.

$$
E I C V=\sum_{i=1}^{n}(D A * F E * P C G)(\text { E. 2) }
$$

Donde:

EICV: resultado de la evaluación del impacto de ciclo de vida.

i: etapas del sistema del producto bajo estudio.

$D A$ : datos de actividad, son una medida cuantitativa de la actividad que produce una emisión o remoción de gases de efecto invernadero (INTE/ISO, 2006).

$F E$ : factores de emisión de un gas de efecto invernadero en específico $\left(\mathrm{CO}_{2}, \mathrm{CH}_{4} \mathrm{y}\right.$ $\mathrm{N}_{2} \mathrm{O}$ ), relaciona los datos de actividad con las emisiones o remociones de carbono. PCG: potenciales de calentamiento, factores que describen la fuerza de radiación de una unidad en relación con la unidad de $\mathrm{CO}_{2}$ en un periodo de tiempo determinado (INTE/ISO, 2006).

Para la EICV se utilizaron factores de emisiones nacionales (IMN, 2015) y factores por defecto (IPCC, 2006) (Cuadro 1). El indicador de categoría utilizado fue el equivalente de dióxido de carbono $\left(\mathrm{CO}_{2}\right.$-eq). El factor de caracterización empleado fue el PCG para un horizonte del tiempo de 100 años $\left(\mathrm{PCG}_{100}\right)$ según el tipo de GEI: $\mathrm{CO}_{2}=1, \mathrm{CH}_{4}=28$ y $\mathrm{N}_{2} \mathrm{O}=265$ (Myhre et al., 2013). Las emisiones biogénicas de $\mathrm{CO}_{2}$ por combustión y descomposición de la biomasa no fueron tomadas en cuenta en la EICV; únicamente se reportan en el ICV con el propósito de incrementar el conocimiento y el debate acerca de las mismas.

Las emisiones de $\mathrm{CH}_{4}$ y $\mathrm{N}_{2} \mathrm{O}$ de la combustión de biomasa no se incluyen en la EICV, pues se determinó que los datos de entrada de esta fuente presentan una incertidumbre muy alta. El carbono almacenado en el producto se incluyó de forma separada.

\section{Resultados y discusión}

De acuerdo con los resultados, los sistemas de producto de tarimas en la región se pueden clasificar según el tipo de combustible utilizado en el tratamiento fitosanitario en: i) sistema de producto con biomasa en el tratamiento fitosanitario (SP-Bio) y ii) sistema de producto con gas licuado de petróleo (SP-GLP). Se estimó que cerca del 76 \% de las tarimas se elaboran bajo el SP-Bio y un $24 \%$ bajo el SP-GLP, cifras calculadas a través de observaciones de campo y de las entrevistas realizadas. En el Cuadro 3 se presentan los resultados del ICV.

\begin{tabular}{|c|c|c|}
\hline 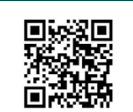 & (c) () (\$) & 40 \\
\hline
\end{tabular}




\section{Revista de CIENCIAS AMBIENTALES Tropical Journal of Environmental Sciences}

Revista de Ciencias Ambientales (Trop J Environ Sci) e-ISSN: 2215-3896 (Enero-Junio, 2021) . Vol 55(1): 32-51 DOI: https://doi.org/10.15359/rca.55-1.2 Open Access: www.revistas.una.ac.cr/ambientales e-mail: revista.ambientales@una.ac.cr Solano-Salmerón J., Fonseca-González W., Ugalde-Alfaro S. y Alice-Guier F.

\subsection{Evaluación del impacto de ciclo de vida (EICV)}

La EICV sobre el cambio climático de las tarimas elaboradas el con SP-Bio es de $2.86 \mathrm{~kg}$ de $\mathrm{CO}_{2}$-eq tarima ${ }^{-1}$ y para el SP-GLP de $3.07 \mathrm{~kg} \mathrm{CO}_{2}$-eq tarima ${ }^{-1}$ (Cuadro 3). Estos resultados son similares a los obtenidos por Niero et al. (2014) y García et al. (2016). En un estudio realizado a una empresa italiana utilizando como producto de referencia una tarima no reversible de cuatro entradas, Niero et al. (2014) obtuvieron valores de $2.27 \mathrm{~kg} \mathrm{CO}_{2}$-eq tarima ${ }^{-1}$; y cifras que oscilan entre 1.57-3.17 $\mathrm{kg} \mathrm{CO}_{2}$-eq tarima ${ }^{-1}$ al aplicar modelos paramétricos.

Por su parte, García et al. (2016), en una investigación realizada en Cataluña-España, obtuvieron valores de $2.12 \mathrm{~kg} \mathrm{CO}_{2}$-eq para una tarima en diseño europeo, cuyos límites del sistema de producto comprendió desde la extracción de las materias primas hasta la puerta de fábrica. Otros estudios sobre ACV en tarimas de madera reportan valores superiores, por ejemplo, Anil (2010) cita $20.05 \mathrm{~kg} \mathrm{CO}_{2}$-eq tarima ${ }^{-1}$ en Estados Unidos y Solano (2018) menciona 6.87-10 kg $\mathrm{CO}_{2}$-eq tarima ${ }^{-1}$ en Costa Rica tomando en cuenta las emisiones biogénicas de $\mathrm{CO}_{2}$ en los sistemas evaluados.

En la segunda unidad funcional del estudio (un metro cúbico de madera aserrada, destinado al mismo uso), los resultados muestran valores de $107.12 \mathrm{~kg} \mathrm{CO}_{2}$-eq m${ }^{-3}$ para el SPBio y $115.05 \mathrm{~kg} \mathrm{CO}_{2}$-eq m${ }^{-3} \mathrm{el} \mathrm{SP-GLP} \mathrm{(Cuadro} \mathrm{3),} \mathrm{cifras} \mathrm{muy} \mathrm{cercanas} \mathrm{a} \mathrm{las} \mathrm{encontradas}$ por Puettmann, Oneil y Bergman (2013) para madera seca y cepillada en la región noreste y centro norte de Estados Unidos $\left(92.9 \mathrm{~kg} \mathrm{CO}_{2}-\mathrm{eq} \mathrm{m}^{-3}\right)$ y a las reportadas por Martínez y Berdasco (2015) para vigas de madera en la región norte de España (95.2 $\mathrm{kg} \mathrm{CO}_{2}$-eq m${ }^{-3}$ ). Los resultados obtenidos muestran que más del $93.1 \%$ del impacto se debe a emisiones de $\mathrm{CO}_{2}$, mientras que el $6,9 \%$ corresponden a $\mathrm{CH}_{4}$ y $\mathrm{N}_{2} \mathrm{O}$.

La comparación de los resultados obtenidos en esta investigación con los reportados en la bibliografía permitió concluir que son coherentes, lo que podría ser un buen indicador de la calidad de los datos primarios utilizados. Además, la cercanía de los resultados con respecto a otros estudios parece indicar que no existen mayores diferencias al haber realizado los cálculos sin un programa especializado (p.ej., SimaPro, GaBi, Umberto, entre otros). No obstante, se debe tener en cuenta que la comparación de resultados de ACV se debe interpretar con precaución, ya que existen diferencias metodológicas importantes entre los estudios, lo cual, ya se ha demostrado, puede conllevar a diferencias importantes en los resultados (De Rosa, Pizzol y Schmidt, 2017; Klein, Wolf, Schulz y Weber, 2015).

El transporte de madera rolliza, la distribución de las tarimas y el aserrío de la madera, son las etapas que generan mayor impacto y representan en conjunto entre el 73-78 \% del impacto de ciclo de vida según el sistema del producto. En ambos casos, el transporte de madera rolliza es la etapa más intensa en emisiones de GEI (Figura $4 \mathbf{a} \mathbf{y} \mathbf{4 b}$ ) con valores de $1.61 \mathrm{~kg} \mathrm{CO}_{2}$-eq tarima ${ }^{-1}$ y $43.22 \mathrm{~kg} \mathrm{CO}_{2}$-eq m $\mathrm{m}^{-3}$. Con respecto a las fuentes de $\mathrm{GEI}$, la combustión de diésel es la de mayor magnitud en todas las etapas del ciclo de vida, con valores de $2.31 \mathrm{~kg} \mathrm{CO}_{2}$-eq tarima-1 y $86.57 \mathrm{~kg} \mathrm{CO}_{2}$-eq m${ }^{-3}$, lo que representa cerca del $81 \%$ del impacto en el SP-Bio y $75 \%$ en el SP-GLP (Figura $4 c$ y $4 d$ ).

\begin{tabular}{|c|c|c|c|c|c|}
\hline 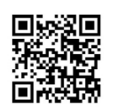 & (c) (i) () () & $\overbrace{\text { AMBERTALIES }}$ & 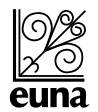 & 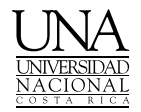 & 41 \\
\hline
\end{tabular}




\section{Revista de CIENCIAS AMBIENTALES Tropical Journal of Environmental Sciences}

Revista de Ciencias Ambientales (Trop J Environ Sci) e-ISSN: 2215-3896

(Enero-Junio, 2021) . Vol 55(1): 32-51

DOI: https://doi.org/10.15359/rca.55-1.2 Open Access: www.revistas.una.ac.cr/ambientales e-mail: revista.ambientales@una.ac.cr Solano-Salmerón J., Fonseca-González W., Ugalde-Alfaro S. y Alice-Guier F.

Cuadro 3. Resultados del inventario del ciclo de vida (ICV) según el sistema del producto para la región Huetar Norte, 2017.

Table 3. Results of the Life Cycle Inventory (LCI) according to the product system for the Huetar Norte Region, 2017.

\begin{tabular}{|c|c|c|c|c|}
\hline \multirow{3}{*}{ Descripción } & \multicolumn{4}{|c|}{ Sistema del producto } \\
\hline & \multicolumn{2}{|c|}{ Tarima } & \multicolumn{2}{|c|}{$\mathbf{m}^{3}$} \\
\hline & SP-Bio & SP-GLP & SP-Bio & SP-GLP \\
\hline \multicolumn{5}{|l|}{ Materia prima } \\
\hline Madera $\left(\mathrm{m}^{3}\right)$ & 0.026 & 0.026 & 1.00 & 1.00 \\
\hline Acero (clavos); kg & 0.201 & 0.201 & 7.62 & 7.62 \\
\hline \multicolumn{5}{|l|}{ Energía } \\
\hline Diésel & $\begin{array}{l}0.851 \mathrm{~L}^{\mathrm{a}} \\
36.59 \mathrm{MJ}^{\mathrm{b}}\end{array}$ & $\begin{array}{l}0.851 \mathrm{~L} \\
36.59 \mathrm{MJ}\end{array}$ & $\begin{array}{l}31.90 \mathrm{~L} \\
1371.78 \mathrm{MJ}\end{array}$ & $\begin{array}{l}31.90 \mathrm{~L} \\
1371.78 \mathrm{MJ}\end{array}$ \\
\hline Gasolina & $\begin{array}{l}0.035 \mathrm{~L} \\
1.57 \mathrm{MJ}\end{array}$ & $\begin{array}{l}0.035 \mathrm{~L} \\
1.57 \mathrm{MJ}\end{array}$ & $\begin{array}{l}1.32 \mathrm{~L} \\
58.44 \mathrm{MJ}\end{array}$ & $\begin{array}{l}1.32 \mathrm{~L} \\
58.44 \mathrm{MJ}\end{array}$ \\
\hline GLP & $\mathrm{NA}^{\mathrm{a}}$ & $\begin{array}{l}0.130 \mathrm{~L} \\
6.16 \mathrm{MJ}\end{array}$ & $\mathrm{NA}$ & $\begin{array}{l}4.92 \mathrm{~L} \\
232.95 \mathrm{MJ}\end{array}$ \\
\hline Electricidad (kWh) & 0.751 & 0.751 & 28.42 & 28.42 \\
\hline Biomasa & $\begin{array}{l}1.76 \mathrm{~kg} \\
28.32 \mathrm{MJ}\end{array}$ & NA & $\begin{array}{l}66.70 \mathrm{~kg} \\
1071.9 \mathrm{MJ}\end{array}$ & NA \\
\hline \multicolumn{5}{|l|}{ Entradas auxiliares } \\
\hline Fertilizante (kg) & 0.032 & 0.032 & 1.18 & 1.18 \\
\hline Úrea $(\mathrm{kg})$ & 0.008 & 0.008 & 0.296 & 0.296 \\
\hline $\mathrm{N}$ sintético $(\mathrm{kg})$ & 0.004 & 0.004 & 0.138 & 0.138 \\
\hline Carbonato de calcio $\left(\mathrm{CaCO}_{3}\right) ; \mathrm{kg}$ & 0.017 & 0.017 & 0.631 & 0.631 \\
\hline Carbonato de magnesio $\left(\mathrm{MgCO}_{3}\right) ; \mathrm{kg}$ & $4.7 \times 10^{-8}$ & $4.7 \times 10^{-8}$ & $1.7 \times 10^{-6}$ & $1.7 \times 10^{-6}$ \\
\hline Óxido de calcio $(\mathrm{CaO}) ; \mathrm{kg}$ & 0.00305 & 0.00305 & 0.114 & 0.114 \\
\hline Lubricantes & $\begin{array}{l}0.055 \mathrm{~L} \\
2.21 \mathrm{MJ}\end{array}$ & $\begin{array}{l}0.055 \mathrm{~L} \\
2.21 \mathrm{MJ}\end{array}$ & $\begin{array}{l}2.07 \mathrm{~L} \\
83.36 \mathrm{MJ}\end{array}$ & $\begin{array}{l}2.07 \mathrm{~L} \\
83.36 \mathrm{MJ}\end{array}$ \\
\hline \multicolumn{5}{|l|}{ Emisiones (kg) } \\
\hline $\mathrm{CO}_{2}($ fósil $)$ & 2.82 & 3.03 & 105.77 & 113.69 \\
\hline $\mathrm{CO}_{2}$ (biogénico, combustión biomasa $)^{\mathrm{d}}$ & 3.17 & NA & 120.05 & NA \\
\hline $\mathrm{CH}_{4}$ (fósil) & 0.00026 & 0.00027 & 0.0099 & 0.01000 \\
\hline $\mathrm{CH}_{4}$ (biogénico, combustión biomasa) ${ }^{\mathrm{d}}$ & 0.00850 & NA & 0.3216 & NA \\
\hline $\mathrm{N}_{2} \mathrm{O}$ (fósil) & 0.00011 & 0.00011 & 0.00406 & 0.00407 \\
\hline $\mathrm{N}_{2} \mathrm{O}$ (biogénico, combustión biomasa) ${ }^{d}$ & 0.00011 & NA & 0.00429 & NA \\
\hline \multicolumn{5}{|l|}{ Desechos $(\mathbf{k g})$} \\
\hline Biomasa (ecosistema) & 14.37 & 14.37 & 544.0 & 544.0 \\
\hline Biomasa (aserrío) & 0.125 & 0.125 & 4.75 & 4.75 \\
\hline \multicolumn{5}{|l|}{ Co-producto $(\mathbf{k g})$} \\
\hline Biomasa (residual de aserrío, usos múltiples) & 2.84 & 2.84 & 107.3 & 107.3 \\
\hline \multicolumn{5}{|l|}{ Reservorio de $\mathrm{C}(\mathrm{kg} \mathrm{CO}-$-eq) } \\
\hline $\mathrm{C}$ en producto & 19.0 & 19.0 & 718.5 & 718.5 \\
\hline
\end{tabular}

${ }^{\mathrm{a}}$ L: litros, ${ }^{\mathrm{b}} \mathrm{MJ}$ : Megajulios, ${ }^{\mathrm{c}} \mathrm{NA}$ : no aplica, ${ }^{\mathrm{d}}$ emisiones no contempladas en la EICV.

(cc)(




\section{Revista de CIENCIAS AMBIENTALES Tropical Journal of Environmental Sciences}

Revista de Ciencias Ambientales (Trop J Environ Sci) e-ISSN: 2215-3896 (Enero-Junio, 2021) . Vol 55(1): 32-51 DOI: https://doi.org/10.15359/rca.55-1.2 Open Access: www.revistas.una.ac.cr/ambientales e-mail: revista.ambientales@una.ac.cr Solano-Salmerón J., Fonseca-González W., Ugalde-Alfaro S. y Alice-Guier F.

\subsection{Carbono en producto}

En promedio, una tarima destinada a la manipulación y el trasiego de productos agrícolas de exportación almacena $19 \mathrm{~kg} \mathrm{CO}_{2}$-eq tarima ${ }^{-1}$ (Cuadro 3) con valores que oscila entre 13.5-27.2 $\mathrm{kg} \mathrm{CO}_{2}$-eq tarima ${ }^{-1}$. Los valores más bajos corresponden a tarimas fabricadas en su totalidad con melina (G. arborea) y los valores más altos a aquellas fabricadas en mayor o menor medida con otras especies como: teca (Tectona grandis), amarillón (Terminalia amazonia), pilón (Hyeronima alchorneoides). Lo anterior se debe a las diferencias encontradas en la densidad de la madera y la fracción de carbono de cada una de las especies. En la segunda unidad funcional (un $\mathrm{m}^{3}$ de ma-

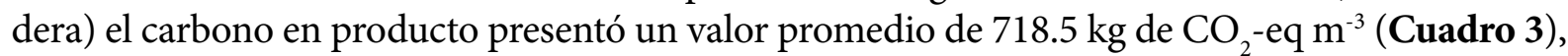
con un rango entre 510.4 y $1031.2 \mathrm{~kg} \mathrm{CO}_{2}$-eq m$~^{-3}$. En el caso del carbono en tarimas los resultados son considerablemente mayores a los reportados por García et al. (2016) quienes obtuvieron un valor de $8.2{\mathrm{~kg} \mathrm{de} \mathrm{CO}_{2} \text {-eq tarima }}^{-1}$, mientras que el carbono almacenado por $\mathrm{m}^{3}$ de madera es similar a lo encontrado por Puettmann, Oneil y Bergman (2013) y Klein et al. (2015).

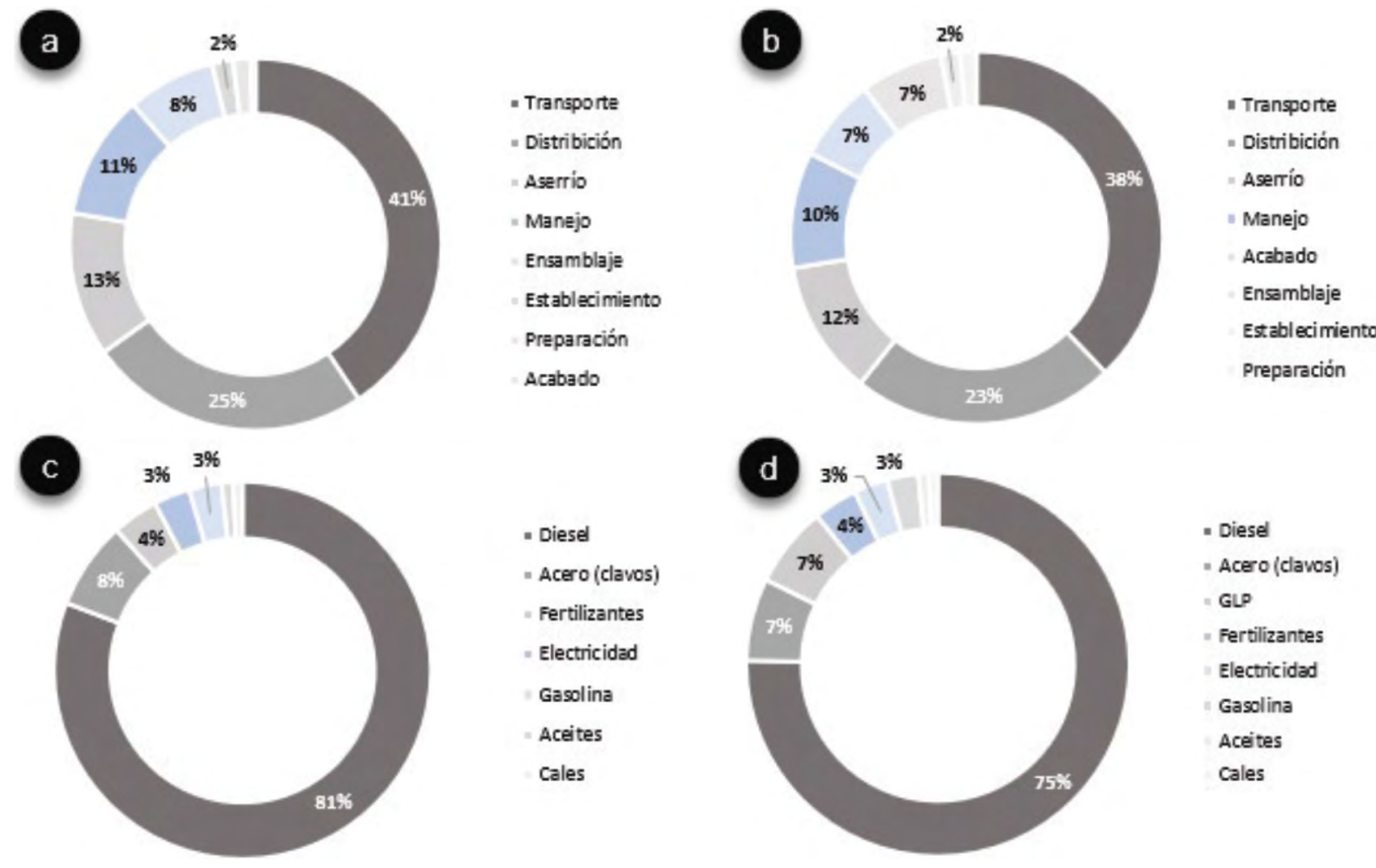

Figura 4. Distribución porcentual de las emisiones de GEI por etapa de ciclo de vida (a: SP-Bio, b: SP-GLP) y por fuente de emisión (c: SP-Bio, d: SP-GLP) para las tarimas de madera en la región Huetar Norte, Costa Rica entre 2015-2017. Figure 4. Percentage distribution of GHG emissions by life cycle stage (a: SP-Bio, b: SP-GLP) and by emission source (c: SP-Bio, d: SP-GLP) for pallets of wood in the Huetar Norte region, Costa Rica between 2015-2017.

\begin{tabular}{|c|c|c|c|c|c|}
\hline 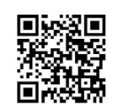 & (c) (1) $\$(0)$ & $\overbrace{\text { AMBENETIES }}$ & $\frac{1 \%}{\text { euna }}$ & 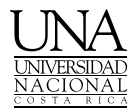 & 43 \\
\hline
\end{tabular}




\section{Revista de CIENCIAS AMBIENTALES Tropical Journal of Environmental Sciences}

Revista de Ciencias Ambientales (Trop J Environ Sci) e-ISSN: 2215-3896 (Enero-Junio, 2021) . Vol 55(1): 32-51 DOI: https://doi.org/10.15359/rca.55-1.2 Open Access: www.revistas.una.ac.cr/ambientales e-mail: revista.ambientales@una.ac.cr Solano-Salmerón J., Fonseca-González W., Ugalde-Alfaro S. y Alice-Guier F.

\subsection{Oportunidades de mejora del desempeño ambiental del producto}

Las oportunidades de mejora del desempeño ambiental de las tarimas se deben enfocar, en primer lugar, en el transporte de madera rolliza, debido a que las medidas de mitigación que se tomen en esta etapa pueden generar un mayor margen de reducción de GEI en comparación con las demás etapas del ciclo de vida.

En la región, existen dos elementos claves para la mitigación del impacto sobre el cambio climático asociados al transporte de madera rolliza que se deben priorizar. En primer lugar, el consumo de combustible por kilómetro recorrido, debido a que los vehículos utilizados en estas etapas se caracterizan por ser de tecnología antigua y presentan un estado regular de funcionamiento, lo que implica que sean poco eficientes y, en consecuencia, generan más emisiones por kilómetro recorrido. El segundo elemento clave que se debe tomar en cuenta para la mitigación del impacto sobre el cambio climático es la distancia de acarreo de la madera. Esta se refiere a la distancia entre las plantaciones forestales y la planta de aserrío, la cual está principalmente relacionada al aseguramiento sostenible del recurso forestal en la región para evitar que la madera deba ser transportada desde regiones más lejanas. Por ello, los incentivos y las políticas públicas orientados a fomentar la producción forestal en la región parecen ser muy importantes en la mitigación del impacto sobre el cambio climático de las tarimas de madera. Además, intervienen elementos del manejo de la logística de las empresas que se podrían profundizar con el fin de identificar oportunidades de mejora del desempeño ambiental del producto. Otra oportunidad de mejora ambiental, en términos de cambio climático, es la posible sustitución del diésel convencional por biodiésel u otros combustibles con menor huella de carbono.

\section{Conclusiones}

Los resultados obtenidos en esta investigación son coherentes con los logrados en otras investigaciones reportadas previamente en la bibliografía, lo que indica que no existen mayores diferencias al haber realizado los cálculos sin un programa especializado en ACV. El transporte de madera rolliza es la etapa del ciclo de vida que libera mayores emisiones de GEI, debido a la combustión de diésel. Las oportunidades de mejora del desempeño ambiental del producto se deben enfocar en primera instancia en el transporte de la madera rolliza, principalmente en mejorar la eficiencia en el consumo de combustible de los vehículos de carga y en reducir la distancia de desplazamiento entre las plantaciones forestales y la planta de aserrío.

\section{5. Ética y conflicto de intereses}

Las personas autoras declaran que han cumplido totalmente todos los requisitos éticos y legales pertinentes, tanto durante el estudio como en la producción del manuscrito; que no hay conflicto de interés de ningún tipo; que todas las fuentes financieras se mencionan completa y claramente en la sección de agradecimientos, y que están totalmente de acuerdo con la versión final editada del artículo.

\begin{tabular}{|c|c|c|c|c|c|}
\hline 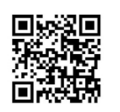 & (c) (i) () () & $\overbrace{\text { AMBERTALIES }}$ & 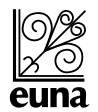 & 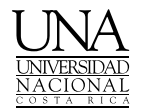 & 44 \\
\hline
\end{tabular}




\section{Revista de \\ CIENCIAS AMBIENTALES \\ Tropical Journal of Environmental Sciences}

Revista de Ciencias Ambientales (Trop J Environ Sci) e-ISSN: $2215-3896$ (Enero-Junio, 2021) . Vol 55(1): 32-51 DOI: https://doi.org/10.15359/rca.55-1.2 Open Access: www.revistas.una.ac.cr/ambientales e-mail: revista.ambientales@una.ac.cr Solano-Salmerón J., Fonseca-González W., Ugalde-Alfaro S. y Alice-Guier F.

\section{Agradecimientos}

Al proyecto "Manejo del Cambio Climático a través del Sector Forestal en Costa Rica" de la Escuela de Ciencias Ambientales de la Universidad Nacional (EDECA-UNA) de Costa Rica, por darme la oportunidad de incursionar en el tema, por la asesoría, el seguimiento y el apoyo técnico y de logística brindado. A la Oficina Nacional Forestal de Costa Rica por la asesoría, el seguimiento y el apoyo técnico y financiero. Además, agradecer a la Dirección de Extensión y a la Dirección de Investigación de la Universidad Nacional (UNA), por el apoyo financiero brindado por medio del Fondo para el Fortalecimiento de las Capacidades Estudiantiles (FOCAES). Finalmente, agradecer a la Revista y a las personas revisoras anónimas por las útiles observaciones al manuscrito.

\section{Referencias}

Anil, S. K. (2010). Environmental analysis of pallets using life cycle analysis and multi-objective dynamic programming [Tesis Mg.sc.]. The Pennsylvania State University.

Blanco, M. L., Carpio, I., Muñoz, F. (2005). Fichas técnicas de veinte especies maderables de importancia en Costa Rica. UCR.

CCT (Centro Científico Tropical) y MAG (Ministerio de Agricultura y Ganadería). (2004). Zonas de vida de Costa Rica según la clasificación de zonas de vida del mundo de L.R. Holdrigde; Atlas de Costa Rica 2014 (shape). San José, C.R.

Costa Rica/MINAET (Ministerio de Ambiente, Energía y Telecomunicaciones). (2009). Estrategia nacional de cambio climático. Editorial Calderón y Alvarado.

Cubero, J. y Rojas, S. (1999). Fijación de carbono en plantaciones de melina (Gmelina arborea Roxb.), teca (Tectona grandis L.f) y pochote (Bombacopsis quinata Jacq.) en los cantones de Hojancha y Nicoya, Guanacaste, Costa Rica. [Tesis de licenciatura]. Universidad Nacional de Costa Rica.

De Rosa, M., Pizzol, M. y Schmidt, J. (2017). How methodological choices affect LCA climate impact results: the case of structural timber. The International Journal of Life Cycle Assessment, 23(1). https://www.researchgate.net/publication/316251913. https://doi.org/10.1007/ s11367-017-1312-0

Elia, V. y Grazia, M. (2015). Designing an effective closed loop system for pallet management. Int. J. Production Economics, 170, 730-740. https://doi.org/10.1016/j.ijpe.2015.05.030

FAO (Food and Agriculture Organization of the United Nations). (2006a). Livestock's long shadow: environmental issues and options (H. Steinfeld; P. Gerber; T. Wassenaar; V. Castel; M. Rosales; C. de Haan, I. T. Rome, Eds.). FAO.

\begin{tabular}{|c|c|c|c|c|c|}
\hline 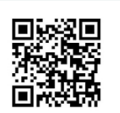 & (c) (i) () & $\underbrace{}_{\text {AMBIFNAIIES }}$ & $\begin{array}{l}\frac{9 \%}{2} \\
\frac{2}{2} \\
\text { euna }\end{array}$ & 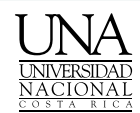 & 45 \\
\hline
\end{tabular}




\section{Revista de \\ CIENCIAS AMBIENTALES \\ Tropical Journal of Environmental Sciences}

Revista de Ciencias Ambientales (Trop J Environ Sci) e-ISSN: $2215-3896$ (Enero-Junio, 2021) . Vol 55(1): 32-51 DOI: https://doi.org/10.15359/rca.55-1.2 Open Access: www.revistas.una.ac.cr/ambientales e-mail: revista.ambientales@una.ac.cr Solano-Salmerón J., Fonseca-González W., Ugalde-Alfaro S. y Alice-Guier F.

FAO (Food and Agriculture Organization of the United Nations). (2006b). Normas internacionales para medidas fitosanitarias: NIMF-15 directrices para reglamentar el embalaje de madera utilizado en el comercio internacional (2002). FAO.

FAO (Food and Agriculture Organization of the United Nations). (2010). Impact of the global forest industry on atmospheric greenhouse gases. Autor.

Fonseca, W. (2009). Restauración forestal y almacenamiento de carbono en el trópico húmedo (zona caribe de Costa Rica) [Tesis de doctorado]. Universidad de Alcalá.

García, L., Farreny, R., Navarro, P. y Boschmonart, J. (2016). Life cycle assessment of a coniferous Wood supply chain for pallet production in Catalonia, Spain. Journal of Cleaner Production, 137,178-188. https://doi.org/10.1016/j.jclepro.2016.07.032

Guevara, M. y Murillo, O. (2009). Costos y rendimientos de ocho tipos de poda en plantaciones jóvenes de Acacia mangium Willd en la zona norte de Costa Rica. Revista Forestal Kurú, 6(17), 1-7.

Heinimann, H. R. (2012). Life cycle assessment (LCA) in forestry - state and perspectives. Croat. Croatian Journal of Forest Engineering, 33(2), 357-372.

IMN (Instituto Meteorológico Nacional). (2014). Clima en Costa Rica: Clima de Costa Rica y variabilidad climática. https://www.imn.ac.cr/clima-en-costa-rica

IMN (Instituto Meteorológico Nacional). (2015). Factores de emisión de gases de efecto invernadero ( $5^{\text {ta }}$ ed.). Autor.

INTE/ISO. (2006). Gases de efecto invernadero - parte 1: Especificaciones con orientación, a nivel de las organizaciones, para la cuantificación y el informe de las emisiones y remociones de gases de efecto invernadero. INTECO.

INTE/ISO. (2007a). Norma 14040. Gestión ambiental, análisis del ciclo de vida: Principios y marco de referencia. INTECO.

INTE/ISO. (2007b). Norma 14044. Gestión ambiental, análisis del ciclo de vida: Requisitos y directrices. INTECO.

IPCC (Grupo Intergubernamental de Expertos sobre el Cambio climático). (2006). En H. S. Eggleston, L. Buendia, K. Miwa, T. Ngara, K. Tanabe (Eds.), Directrices del IPCC de 2006 para los inventarios nacionales de gases de efecto invernadero. IPPC.

IPCC (Grupo Intergubernamental de Expertos sobre el Cambio climático). (2014). Climate Change 2014: Mitigation of Climate Change. Contribution of Working Group III to the Fifth Assessment. Report of the Intergovernmental Panel on Climate Change (O. Edenhofer, R. Pichs-Madruga, Y. Sokona, E. Farahani, S. Kadner, K. Seyboth, A. Adler, I. Baum, S. Brunner, P.

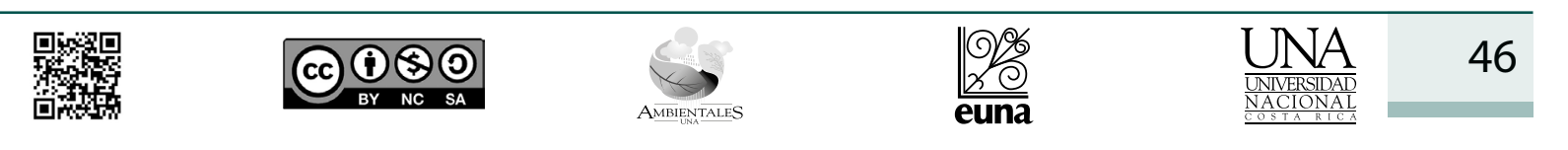




\section{Revista de \\ CIENCIAS AMBIENTALES \\ Tropical Journal of Environmental Sciences}

Revista de Ciencias Ambientales (Trop J Environ Sci) e-ISSN: $2215-3896$ (Enero-Junio, 2021) . Vol 55(1): 32-51 DOI: https://doi.org/10.15359/rca.55-1.2 Open Access: www.revistas.una.ac.cr/ambientales e-mail: revista.ambientales@una.ac.cr Solano-Salmerón J., Fonseca-González W., Ugalde-Alfaro S. y Alice-Guier F.

Eickemeier, B. Kriemann, J. Savolainen, S. Schlömer, C. von Stechow, T. Zwickel, J.C. Minx., Eds.). Cambridge University Press.

Klein, D., Wolf, C., Schulz, C. y Weber, G. (2015). 20 years of life cycle assessment (LCA) in the forestry sector: State of the art and a methodical proposal for the LCA of forest production. The International Journal of Life Cycle Assessment, 20, 556-575. https://www.researchgate.net/ publication/273294486. https://doi.org/10.1007/s11367-015-0847-1

Life Cycle Initiative, F. R. (2017). What is Life Cycle Thinking? https://www.lifecycleinitiative. org/starting-life-cycle-thinking/what-is-life-cycle-thinking/

Louman, B., Gómez, M. y Navarro, G. (2006). Determinación de costos, productividad y rendimientos del aprovechamiento forestal. En L. Orozco, C. Brumér, D. Quirós (Eds.), Aprovechamiento de impacto reducido en bosques latifoliados húmedos tropicales (pp. 278-312). CATIE.

Martínez, C. y Berdasco, L. (2015). Carbon footprint of sawn timber products of Castanea sativa Mill., in the north of Spain. Journal of Cleaner Production,102,127-135. https://doi.org/10.1016/j.jclepro.2015.05.004

Mazeika, A. (2011). Environmental impact analysis of alternative pallet management system [Tesis de maestría]. Rochester Institute of Technology.

Moya, R., Muñoz, F., Salas, C., Berrocal, A., Leandro, L., Esquivel, E. (2010). Tecnología de maderas de plantaciones forestales: Fichas técnicas. Revista Forestal Mesoamericana Kurú, 7(1819), 87-101. https://revistas.tec.ac.cr/index.php/kuru/article/view/383/2355

Myhre, G., Shindel, D., Bréon, F.M., Collins, W., Fuglestvedt, J., Huang, J., Koch, D., Lamarque J. F., Lee, D., Mendoza, B., Nakajima, T., Robock. A., Stephens, G., Takemura, T. y Zhang, H. (2013). Anthropogenic and Natural Radiative Forcing. In Climate Change 2013: The Physical Science Basis. Contribution of Working Group I to the Fifth Assessment Report of the Intergovernmental Panel on Climate Change. Eds: Stocker, T. F., D. Qin, G.-K. Plattner, M. Tignor, S. K. Allen, J. Boschung, A. Nauels, Y. Xia, V. Bex and P. M. Midgley. Cambridge University Press.

Niero, M., Di Felice, F. Ren, J. (2014). How can a life cycle inventory parametric model streamline life cycle assessment in the wooden pallet sector? The International Journal of Life Cycle Assessment, 19, 901-918. https://doi.org/10.1007/s11367-014-0705-6

ONF (Oficina Nacional Forestal). (2018). Usos y aportes de la madera en Costa Rica. Estadísticas 2017 y precios 2018 (B. Alfonso, S. Ugalde, Eds.) ONF.

Platt, B., Hyde, J. (1997). Sustaining businesses \& Jobs through pallet repair \& reuse. Institute for Local Self-Reliance.

\begin{tabular}{|c|c|c|c|c|c|}
\hline 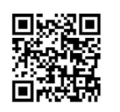 & (c) $\underset{\mathrm{BY}}{(1)}(0)$ & $\underset{\text { AMBENETILES }}{3}$ & $\begin{array}{l}\frac{O O}{2} \\
\frac{2}{2} \\
\text { euna }\end{array}$ & 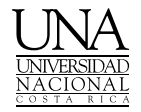 & 47 \\
\hline
\end{tabular}




\section{Revista de CIENCIAS AMBIENTALES Tropical Journal of Environmental Sciences}

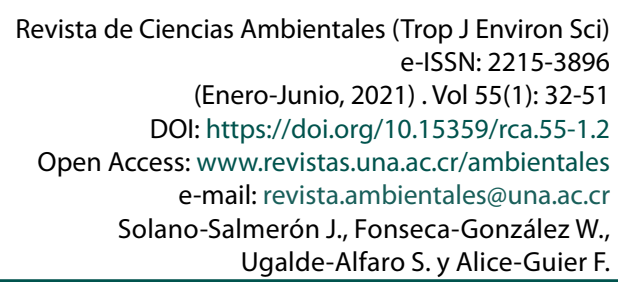
Ugalde-Alfaro S. y Alice-Guier F.

Puettmann, M., Oneil, E. y Bergman, R. (2013). Cradle to gate life cycle assessment of softwood lumber production from the Northeast-North Central. CORRIM, University of Washington.

Reiche, C. (1989). Manual para determinar rendimientos y costos de faena de producción de los sistemas de árboles uso múltiple. CATIE/ROCAD.

Sage, L. F. y Quirós, R. (2001). Proyección del volumen de madera para aserrío proveniente de las plantaciones de melina y teca y de otras fuentes: Documento técnico preparado como componente del proyecto TCP/COS/006(A) "mercadeo e industrialización de madera proveniente de plantaciones forestales".

SINAC (Sistema Nacional de Áreas de Conservación). (2011). Censo nacional de la industria forestal primaria de Costa Rica (M. Camacho, D. Camacho y F, Monge, Eds.). SIREFOR (Sistema de información de recursos forestales).

SINAC, SIREFOR y MINAE (Sistema Nacional de Áreas de Conservación, Sistema de Información de los Recursos Forestales y Ministerio de Ambiente y Energía). (2013). Reporte estadístico forestal 2013 (M.I. Chavarría Espinoza, M., Castillo Núñez, Eds.). GIZ.

Solano, J. (2018). Impacto potencial sobre el cambio climático de las tarimas de madera elaboradas en la región Huetar Norte de Costa Rica a través de un análisis de ciclo de vida (ACV) [Tesis de licenciatura]. Universidad Nacional, Costa Rica.

\begin{tabular}{|c|c|c|c|c|}
\hline 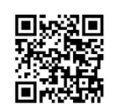 & (c) (i) () () & $\underset{\text { AMBEETILIES }}{3}$ & $\frac{1 \%}{2 \%}$ & 48 \\
\hline
\end{tabular}




\section{Revista de \\ CIENCIAS AMBIENTALES Tropical Journal of Environmental Sciences}

Revista de Ciencias Ambientales (Trop J Environ Sci)

e-ISSN: 2215-3896

(Enero-Junio, 2021) . Vol 55(1): 32-51

DOI: https://doi.org/10.15359/rca.55-1.2

Open Access: www.revistas.una.ac.cr/ambientales

e-mail: revista.ambientales@una.ac.cr

Solano-Salmerón J., Fonseca-González W., Ugalde-Alfaro S. y Alice-Guier F.

\section{Apéndices}

Apéndice 1. Entradas de energía, materias primas, entradas auxiliares y productos intermedios de las etapas del ciclo de vida (CV) de las tarimas de madera elaboradas en la región.

Appendix 1. Energy, raw materials, auxiliary and co-products inputs of the life cycle of wooden pallets in study region.

\begin{tabular}{|c|c|c|c|c|c|}
\hline Etapa del CV & Procesos unitarios & $\begin{array}{c}\text { Fuentes de } \\
\text { energía }\end{array}$ & $\begin{array}{c}\text { Materias } \\
\text { primas }\end{array}$ & $\begin{array}{c}\text { Entradas } \\
\text { auxiliares } \\
\end{array}$ & $\begin{array}{c}\text { Productos } \\
\text { intermedios }\end{array}$ \\
\hline Preparación & $\begin{array}{l}\text { Drenado de aguas, chapea (destronque, } \\
\text { barrido y acordonado) (carrileo), arado, } \\
\text { subsolado, formación de camellones, } \\
\text { encalado, trazado y rodajea }\end{array}$ & $\begin{array}{l}\text { Diésel y } \\
\text { gasolina }\end{array}$ & $\mathrm{NA}^{\mathrm{a}}$ & $\begin{array}{l}\text { Aceites, grasas, } \\
\text { lubricantes, } \\
\text { herbicidas y cal }\end{array}$ & NA \\
\hline Establecimiento & $\begin{array}{l}\text { Ahoyado, fertilización inicial, siembra y } \\
\text { resiembra }\end{array}$ & Gasolina $^{\mathrm{b}}$ & NA & $\begin{array}{l}\text { Fertilizantes, } \\
\text { aceites y } \\
\text { lubricantes }^{c}\end{array}$ & NA \\
\hline \multirow[t]{8}{*}{ Manejo } & Chapea y limpieza de rodajas & $\begin{array}{l}\text { Diésel y } \\
\text { gasolina }^{\mathrm{d}}\end{array}$ & NA & $\begin{array}{l}\text { Aceites, grasas, } \\
\text { lubricantes y } \\
\text { herbicidas }^{\mathrm{e}}\end{array}$ & NA \\
\hline & Segunda y tercera fertilización & NA & NA & Fertilizante & NA \\
\hline & Encalado & NA & NA & Cal & NA \\
\hline & Control de zompopas & NA & NA & Insecticidas & NA \\
\hline & $\begin{array}{l}\text { Curado de las podas, cirugías } \\
\text { fitosanitarias, fumigación posterior a las } \\
\text { podas, fumigación posterior a las cirugías } \\
\text { y fumigación posterior a los raleos }\end{array}$ & NA & NA & $\begin{array}{l}\text { Fungicidas, } \\
\text { aditivos y pintura } \\
\text { de agua }\end{array}$ & NA \\
\hline & $\begin{array}{l}\text { Desinfección de las herramientas, corta } \\
\text { de ramas }\end{array}$ & Gasolina $^{\mathrm{f}}$ & NA & $\begin{array}{l}\text { Aceite, lubricante } \\
\text { y desinfectantes }\end{array}$ & NA \\
\hline & $\begin{array}{l}\text { Volteo, arrastre, apilado, troceo, } \\
\text { saneamiento y cargado }\end{array}$ & $\begin{array}{l}\text { Diésel y } \\
\text { gasolina }\end{array}$ & NA & $\begin{array}{l}\text { Aceites, grasas y } \\
\text { lubricantes }\end{array}$ & NA \\
\hline & $\begin{array}{l}\text { Volteo, arrastre, apilado, troceo, } \\
\text { saneamiento y cargado }\end{array}$ & $\begin{array}{l}\text { Diésel y } \\
\text { gasolina }\end{array}$ & NA & $\begin{array}{l}\text { Aceites, grasas y } \\
\text { lubricantes }\end{array}$ & NA \\
\hline Transporte & Transporte de madera & Diésel & NA & $\begin{array}{l}\text { Aceites, grasas y } \\
\text { lubricantes }\end{array}$ & NA \\
\hline Aserrío & $\begin{array}{l}\text { cubicación, descarga, clasificación de } \\
\text { trozas, troceo, ingreso de la madera a la } \\
\text { planta de transformación, corte primario, } \\
\text { corte secundario, recuperación, despunte, } \\
\text { cabacoteado, elaboración de tacos, } \\
\text { biselado y almacenamiento temporal de } \\
\text { las piezas }\end{array}$ & $\begin{array}{l}\text { Diésel, } \\
\text { gasolina y } \\
\text { electricidad }\end{array}$ & NA & $\begin{array}{l}\text { Aceites, grasas y } \\
\text { lubricantes }\end{array}$ & NA \\
\hline Ensamblaje & $\begin{array}{l}\text { Ingreso de las piezas a sección de } \\
\text { ensamblaje, armado de la tarima, pegado } \\
\text { de las piezas y almacenamiento temporal } \\
\text { de las tarimas }\end{array}$ & Electricidad & Acero & Lubricantes & $\begin{array}{l}\text { Madera } \\
\text { aserrada }\end{array}$ \\
\hline
\end{tabular}

\begin{tabular}{|c|c|c|c|c|c|}
\hline 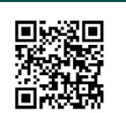 & (c) () & $\overbrace{\text { AMBENTALS }}^{6}$ & $\begin{array}{l}\frac{O \%}{6} \\
\frac{2}{2} \\
\text { euna }\end{array}$ & $\frac{\text { UNA }}{\frac{\text { UNIIERSIDAD }}{\text { NACIONAL }}}$ & 49 \\
\hline
\end{tabular}




\section{Revista de \\ CIENCIAS AMBIENTALES Tropical Journal of Environmental Sciences}

Revista de Ciencias Ambientales (Trop J Environ Sci)

e-ISSN: 2215-3896

(Enero-Junio, 2021) . Vol 55(1): 32-51

DOI: https://doi.org/10.15359/rca.55-1.2

Open Access: www.revistas.una.ac.cr/ambientales

e-mail: revista.ambientales@una.ac.cr Solano-Salmerón J., Fonseca-González W., Ugalde-Alfaro S. y Alice-Guier F.

\begin{tabular}{llllll}
\hline Etapa del CV & \multicolumn{1}{c}{ Procesos unitarios } & $\begin{array}{c}\text { Fuentes de } \\
\text { energía }\end{array}$ & $\begin{array}{c}\text { Materias } \\
\text { primas }\end{array}$ & $\begin{array}{c}\text { Entradas } \\
\text { auxiliares }\end{array}$ & $\begin{array}{c}\text { Productos } \\
\text { intermedios }\end{array}$ \\
\hline Acabado & $\begin{array}{l}\text { Ingreso de las tarimas, tratamiento } \\
\text { térmico, control de la temperatura, } \\
\text { extracción de las tarimas y } \\
\text { almacenamiento temporal }\end{array}$ & $\begin{array}{l}\text { Biomasa, } \\
\text { GLP, diésel y } \\
\text { electricidad }\end{array}$ & NA & Aceites, grasas y & $\begin{array}{l}\text { Tarimas } \\
\text { elaboradas }\end{array}$ \\
& Distribución de las tarimas & Diésel y GLP & NA & Aceites, grasas y & Tarimas \\
Distribución & & & & lubricantes & tratas \\
& & & & &
\end{tabular}

${ }^{a} \mathrm{NA}=$ no aplica.

${ }^{\mathrm{b}}$ La gasolina está asociado al uso de perforadora mecánica, lo cual es una práctica poco frecuente.

${ }^{c}$ Los aceites y lubricantes de esta etapa están asociado al uso de perforadora mecánica, lo cual es una práctica poco frecuente.

d El diésel y la gasolina de este proceso están asociados al uso de chapeador mecánico o moto-guadaña.

${ }^{\text {e}}$ Los aceites, grasas y lubricantes de este proceso están asociados al uso de chapeador mecánico o moto-guadaña.

${ }_{\mathrm{f}}^{\mathrm{f}}$ Asociado al uso de podadoras mecánicas de combustión interna, las cuales son usan con poca frecuencia

Apéndice 2. Procesos unitarios que componen las etapas del ciclo de vida (CV) de las tarimas de madera elaboradas en la región bajo estudio.

Appendix 2. Unit processes of each of the stages of the life cycle of wooden pallets in the study region.

\begin{tabular}{|c|c|c|c|}
\hline Etapa del CV & Actividades & Procesos unitarios & $\begin{array}{c}\text { Total de procesos } \\
\text { unitarios }^{\mathrm{a}}\end{array}$ \\
\hline Preparación & Limpieza del terreno & $\begin{array}{l}\text { Drenado de aguas, chapea (destronque, barrido y } \\
\text { acordonado })^{\mathrm{b}} \text { (carrileo), arado, subsolado, formación } \\
\text { de camellones, encalado, trazado y rodajea }\end{array}$ & 12 \\
\hline Establecimiento & $\begin{array}{l}\text { Siembra y resiembra de los } \\
\text { árboles }\end{array}$ & Ahoyado, fertilización inicial, siembra y resiembra & 4 \\
\hline \multirow[t]{8}{*}{ Manejo } & Control de malezas & Chapea y limpieza de rodajas & $14^{\mathrm{c}}$ \\
\hline & Manejo de la fertilidad & Segunda y tercera fertilización & 2 \\
\hline & Manejo de la acidez & Encalado & $5^{\mathrm{d}}$ \\
\hline & Control de plagas & Control de zompopas (principalmente) & $6^{\mathrm{e}}$ \\
\hline & Control de enfermedades & $\begin{array}{l}\text { Curado de las podas, cirugías fitosanitarias, fumigación } \\
\text { posterior a las podas, fumigación posterior a las } \\
\text { cirugías fitosanitarias y fumigación posterior a los } \\
\text { raleos }\end{array}$ & $17^{\mathrm{f}}$ \\
\hline & Podas & Desinfección de las herramientas, corta de ramas & $6^{\mathrm{g}}$ \\
\hline & Raleos & Volteo, arrastre, apilado, troceo, saneamiento y cargado & $18^{\mathrm{h}}$ \\
\hline & Cosecha & Volteo, arrastre, apilado, troceo, saneamiento y cargado & 6 \\
\hline Transporte & Transporte & Transporte de madera & 1 \\
\hline Aserrío & $\begin{array}{l}\text { Transformación de la } \\
\text { madera }\end{array}$ & $\begin{array}{l}\text { Cubicación, descarga, clasificación de trozas, troceo, } \\
\text { ingreso de la madera a la planta de transformación, } \\
\text { corte primario, corte secundario, recuperación, } \\
\text { despunte, cabacoteado, elaboración de tacos, biselado y } \\
\text { almacenamiento temporal de las piezas }\end{array}$ & 13 \\
\hline
\end{tabular}

\begin{tabular}{|c|c|c|}
\hline 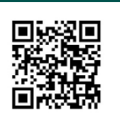 & (c) (1) (2) & 50 \\
\hline
\end{tabular}




\section{Revista de CIENCIAS AMBIENTALES

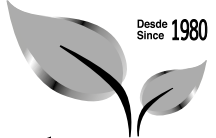 Tropical Journal of Environmental Sciences}

Revista de Ciencias Ambientales (Trop J Environ Sci)

e-ISSN: 2215-3896

(Enero-Junio, 2021) . Vol 55(1): 32-51

DOI: https://doi.org/10.15359/rca.55-1.2

Open Access: www.revistas.una.ac.cr/ambientales

e-mail: revista.ambientales@una.ac.cr Solano-Salmerón J., Fonseca-González W., Ugalde-Alfaro S. y Alice-Guier F.

\footnotetext{
Ensamblaje

Armado de las tarimas

Ingreso de las piezas a sección de ensamblaje, armado

4 de la tarima, pegado de las piezas y almacenamiento temporal

Acabado

Tratamiento fitosanitario

Ingreso de las tarimas, tratamiento térmico, monitoreo y control de la temperatura, extracción de las tarimas y almacenamiento temporal

Distribución Distribución

Cargado, sujetado, cobertura de la carga y distribución

5

4

TOTAL

a Valores para todo el turno de rotación.

${ }^{\mathrm{b}}$ Los valores entre paréntesis representan procesos que desempeñan una misma función.

c Se estima la ejecución de siete chapeas y siete limpiezas de rodajas a lo largo del turno de rotación.

d Algunas empresas aplican aproximadamente una dosis de cal cada dos años, iniciando a partir del año dos, lo que conlleva la aplicación de 5 dosis de cal a lo largo del turno.

e Se estima que en promedio se realizan seis aplicaciones de insecticida para el control preventivo y correctivo de zompopas.

${ }^{\mathrm{f}}$ Considera el curado de las podas, cuatro procesos de cirugía fitosanitaria y 10 fumigaciones.

g Considera que se realizan tres podas en el turno, con herramientas previamente desinfectadas.

${ }^{\mathrm{h}}$ Considera la ejecución de tres raleos, de los cuales se obtiene madera comercial, por lo que en cada raleo se realizan los procesos de volteo, arrastre, apilado, troceo, saneamiento y cargado.
}

(c)

